\title{
CONVULSION AND INSULIN TREATMENT
}

\author{
By Dalton E. Sands, M.R.C.P.Ed., D.P.M. \\ Deputy Physician Superintendent, Banstead Hospital ; Psychiatric Consultant to the L.C.C. ; \\ Associate Psychiatrist to King's College Hospital
}

\section{Convulsion Therapy}

Over 12 years have passed since the first report on convulsion therapy was given by Von Meduna. In that period the treatment has been undergoing the cycle usually imposed on any promising remedy in medicine. Originally used for schizophrenia, it was subsequently tried in a wide field of psychiatric conditions. Now it is becoming recognized as a specific treatment for one form of mental illness, psychotic depression, and as an aid in a number of other conditions. It is significant that despite much controversy and the fundamental repulsiveness of inducing a fit, the method has more than held its place over a fair period of time. A radical change in the usual orientation was needed when for centuries the aim had been to cure rather than provoke fits. Yet the idea of using one potentially noxious agent for the relief of a greater menace is nothing new in other branches of medicine. The essential feature in the method still remains the artificial induction of a major convulsion. There have been many attempts to modify it but so far the only result of note has.been the finding that softening or even elimination of the associated muscular movements has had no untoward effect on the results.

\section{Indications}

Depressions. - Because of the inexactitudes of psychiatric classification, the particular form of depression intended needs a little further definition. The case may be certified or uncertified though it is amongst the former that the more dramatic triumphs are scored. These patients are consistently depressed and their attitude remains much the same whether or not they realize themselves to be observed. They show a diminishing state of awareness, a movement away from reality, and an increasing introspection with a narrowed range of thought. They tend to become completely preoccupied with a few ideas. So severe may the process be that eventually they may be totally inaccessible and in a state of stupor. Some show great agitation but this moves within the narrowed orbit of their thinking. They may be very tense but the tension is dependent on their own conflicts and not, as in the tension of the neuroses, readily influenced by adverse external influences. These depressions are mainly endogenous or constitutional in type. They are seen in the depressed phase of a manic depressive psychosis and in involutional depression with hypochondrasis, nihilism and guilt. The mainly reactive depressions show a similar picture. Occasionally one meets cases precipitated by severe stress, the stress has passed but recovery seems delayed and in these a good treatment result is usually obtained provided it is reasonably certain that the initial stress was the main one. As a rule such exogenous aetiological factors as can be found are inadequate and differ little from common experience.

In addition to the severe forms described, there are many milder depressions of the psychotic group who are incapacitated for work, feel ' run down,' tired, ineffective, having headaches or other bodily symptoms of ill-defined distribution and quality. They are rarely certified but none the less are often subject to much suffering and economic stress if allowed to persist unrecognized. They can present much difficulty in diagnosis since their real nature may be obscured by obsessional, anxious, or hysterical symptoms. Since such patients retain a certain capacity for activity they are liable to succumb to the most feared complication of depressive psychosis. They are responsible for a large proportion of those cases reported by the Coroner as 'Suicide while the balance of the mind was disturbed.'

Schizophrenias. This group of illnesses was the first treated by convulsion therapy, but it was soon apparent that results were inferior to those obtained with certain depressions as already described. In spite of this there are some categories of schizophrenia in which the treatment has value. If insulin therapy is not available, it is worth a trial in cases of less than a year's duration irrespective of subtype, particularly if the cases are acute, of rapid onset, have definite precipitating stresses and possessed of personalities that have been fairly well adjusted to life hitherto. Such patients would in any event carry a better prognosis than most, and some might suggest that . it would be wiser to await the expected remission than to subject a patient to treatment that is not quite free of risk. One has to remember that 
while some features in the schizophrenic are of good import, these are no absolute criteria for a successful outcome. Therefore, to await remission or recovery means taking a considerable chance with the patients' health besides prolonging an illness, that if not terminated in a short time, may have serious occupational, social and economic complications for the patient.

Schizophrenic stupors and confusional states usually respond promptly to convulsion therapy, though liable to relapse if not later treated by insulin comas. The more violent and impulsive phases of the catatonic type are benefited but the effect is largely symptomatic. Some schizophrenias are mixed with depression. If convulsions are used a certain splitting of the psychosis often results since the depression is improved whilst the disordered thinking is little affected.

Hebephrenias, paranoid and simple dementing forms of schizophrenia are not benefited as a rule even by large numbers of fits. Under this heading should certainly be included that sub-group' long known as Dementia Praecox, a term formerly applied to all schizophrenias.

Mania. Unfortunately convulsion treatment does not display the same specific action in mania as it does in depression. It can mitigate an attack and achieve some degree of symptomatic control that is indeed useful enough, but it does not habitually shorten the attack and relapse is frequent. In any event whether the attack is manic or depressive, convulsion therapy confers no immunity on the patient in respect of later attacks.

Paranoia and paraphrenia can be improved if a concentrated series of fits is given, but patients relapse when the confusion and memory disturbance, provoked by the fits, begins to recover. Such types are not normally considered suitable for the treatment and should not be confused with paranoid states that are part of a depressive psychosis and which usually do well.

Generally speaking neuroses are a contraindication to this form of treatment and many may be made worse. No good case has been established for treating the ordinary uncomplicated neurotic patient by this means. The chief difficulty is in diagnosis, since a psychosis of mild severity can be considerably masked by neurotic symptoms. Erroneous conclusions are apt to be drawn on the effect of the treatment in the belief that a neurosis has been benefited. In anxiety states the effect is usually to stimulate more anxiety and tension.

- Hysterias and anxiety neurotic patients are apt to fix their neurotic symptoms on any physical discomfort that may arise out of the treatment and to complain strongly of the same. Temporary dramatic relief can be obtained in hysterical stupors but such conditions can be relieved in less $\frac{\widehat{\Phi}}{\mathbb{Q}}$ drastic ways and in any event psychological $\frac{\varrho}{c}$ treatment is required afterwards.

Obsessional neuroses often benefit while the $\stackrel{\overrightarrow{\vec{S}}}{\vec{P}}$ treatment is proceeding, but relapse when it을 ceases. They are liable to complain early of the memory disturbance and forgetfulness in a way $\frac{\bar{s}}{\vec{D}}$ rarely seen in psychotic patients.

Recently Milligan (1946) has treated chronic neuroses that have proved resistant to other $\overrightarrow{ }$ methods by concentrated fits up to three or four a $?$ day for four to six days followed by others more $\overrightarrow{\vec{\omega}}$ spaced out. The treatment was stated to have $\stackrel{\circ}{\Omega}$ been successful but further experience is needed 8 before conclusions can be drawn. A case of 3 . chronic stammering that had proved very re- i sistant to the more usual lines of treatment such as speech re-education, was benefited by con- $\triangle$ vulsion therapy given every five days though liable to relapse in times of stress (Owen and Stemmerman, 1947).

Contraindications. Depressions associated with $\rightarrow$ organic brain disease or cerebral trauma. Attempts have been made to treat the depressed forms of general paralysis of the insane and even of pre- $\stackrel{\infty}{+}$ senile dementia. Though some transitory relief $\vec{\omega}$ the depression may be achieved the dementin course is accelerated and incontinence may appea for the first time. Similarly depressions that afe likely to have been precipitated by severe cerebral arteriosclerosis should not be treated. In patients with a history of cerebral trauma, it is necessary $\frac{\circ}{\mathbb{Q}}$ to know how far the trauma can be said to have $\varrho$ caused organic change. Where neurological and $\overrightarrow{\overrightarrow{0}}$ electro-encephalographic investigation show that 3 this has occurred, convulsion therapy is to be avoided, though psychotic depressions that have had some definite blow on the head without $\frac{\partial}{3}$ sustaining cortical or other damage, may be treated.

Contraindications are an individual matter in most cases. The gain anticipated has to be $\frac{0}{3}$ balanced against the risks involved with consideration for the hazards of leaving the patient un- $\frac{5}{J}$ treated. For instance, a patient with mitral $\frac{D}{0}$ stenosis associated with strong suicidal tendencies might justify one taking more risk in treatment $N$ than on the same patient not so complicated. N There are few absolute contraindications but $\mathrm{N}$ amongst them should be placed coronary disease, $\omega$ aneurysm of the aorta, peptic ulcers in acute phase and decompensated diseased myocardia.

Systolic blood pressure over 200 and diastolic $\stackrel{\oplus}{\rightarrow}$ pressure over I io should be a bar to treatment if $+\underset{+}{+}$ due to renal rather than emotional causes. In the ábsence of renal lesions the presence of the $\overrightarrow{\mathbb{D}}$ emotional type of high pressure may be confirmed $\stackrel{\rho}{\Phi}$ 
by the injection of 0.2 to $0.4 \mathrm{gm}$. of sodium amytal intravenously, when a fall of from 50 to $80 \mathrm{mgm}$. is usual, and contrasts with the slight change seen in renal cases. Hypertensions of the emotional type can be safely treated by E.C.T. The liability to cerebral haemorrhage is much less than might be supposed. Cases do not appear to have been reported with the possible exception of one who was a poor candidate in any case.

Pulmonary tuberculosis and pregnancy have both been associated with E.C.T., but are best avoided. The former has been discussed. The latter appears to carry little risk if treatment is given before the end of the third month. Two of the author's patients were delivered of normal full time infants after having an average number of fits between the second and third months of pregnancy.

\section{Technique and Management of Convulsion Therapy}

Generally speaking pharmacological methods of inducing a fit have been superseded by electrical. Von Meduna revived the suggestion of Weickhardt, in 1798 , to use camphor injections as the fit provoking agent, but it was uncertain and the more reliable cardiazol (or metrazol) held the field from 1933 to 1937 . Cardiazol is given by the rapid intravenous injection of from 4 to $9 \mathrm{cc}$. according to tolerance. The drawbacks are the necessity for repeated injections two to three times a. week, the highly unpleasant sensations experienced by patients at the onset of the fit and the fact that at least half the patients vomit afterwards even when the treatment is given fasting. Intravenous injections can be avoided by the use of triazol intramuscularly (Mayer-Gross and Walk, 1938), but the resulting fits are uncertain in incidence and a succession of fits sometimes results from 15 minutes to an hour after injection. In practice this modification is difficult to control. Cardiazol is still advocated by some psychiatrists for catatonic stupors or paranoid psychoses, and it is sometimes used in combination with insulin comas for schizophrenia.

Various attempts were made to lessen the unpleasantness of the injections." Many sedatives have been tried for premedication. Cook (1944) advocated the use of hyoscine, while even general anaesthesia was used by Neustatter, et al. (1939). A moderate degree of hypoglycaemia, to the point of stupor or light coma, was used by Sands (i939). Recently Tow (1947) has described a method which involves the injection of sodium amytal as soon as respiration is re-established, with the production of an amnesia for the unpleasant concomitants of cardiazol injections.
From to to 30 seconds after the cardiazol injections a grand mal type of epileptiform attack occurs, and a cry often heralds the onset of the tonic phase. With the onset of this phase the mouth usually opens for a few seconds, and if a gag is not already in position it can be slipped in at that moment. From Io to 15 seconds after the start of the fit the clonic movements begin, and for about 30 seconds steadily increase in amplitude but decrease in frequency. During this phase the pupils dilate and the conjunctivae become engorged. After a few seconds the patient takes a long breath, having been apnoeic since the start of the fit, so that by the finish a fair degree of cyanosis is common. As soon as the clonic stage ceases the head should be tilted to one side and the jaw pushed forward by a finger placed behind the angle on each side. Occasionally the tongue falls back despite this precaution, and tongue forceps should be at hand. If the resumption of respiration is delayed by more than 7 to ro seconds, there should be no delay in applying artificial respiration, and oxygen with 5 per cent. carbon dioxide given through a B.L.B. mask. Respiration is easily resumed after a short application of these remedies. The use of oxygen and artificial respiration need not be routine, but one should always be on the alert to give it especially in those of more advanced years. Immediately after the fit ceases the pulse is often scarcely perceptible, but in most patients it rapidly regains volume though it may remain fast for several minutes. Temporary arrhythmia occurs occasionally but as such has no special significance.

Patients vary greatly in the time required to regain consciousness and so far these variations have not been associated with any special psychiatric types. Some are awake within a minute or two of the end of the fit, while others may need ten minutes to regain consciousness. While the majority recover quickly, some show post-convulsive excitement and may leap out of bed. Most patients are confused in varying degrees for about half an hour to an hour after a fit. Nearly all are easily startled for ten minutes afterwards and will jump violently if anyone unexpectedly appears. It is necessary for staff to be at hand and alert in this period since in the post-convulsive period impulsive movement may be made very suddenly in one hitherto quiet. Some becomè temporarily emotional and dependent, needing reassurance. The patient does not remember the convulsion excepting the onset after the injection as noted. . . ,

Electrical Convulsive Therapy' (E.C.T: as described by Cerletti and Bini (I937) has largely superseded the chemical method. Since loss of consciousness is instantaneous, the patient feels 
nothing of the fit at any stage till consciousness is regained, hence patients objections to the treatment are few. The objectors are chiefly those who by reason of their mental state resist any kind of medical or routine nursing attention. Vomiting is very unusual afterwards and a light meal can be had up to an hour before treatment without fear of this complication. It is much simpler and easier to apply than the pharmacological method.

The machines are designed to supply a certain voltage, usually between 70 and 150 , for a set period of time from 0.1 to I second, using alternating current from electric light mains, or if only direct current is available, by the aid of a transformer. More recent apparatus employs monophasic or diphasic current measured in Joules. After the skin has been cleaned with methylated ether, the current is applied by means of an electrode to each temple just clear of the hair bearing area. The electrodes are covered by pads of gauze or lint soaked in 20 per cent. saline. There are several ways of actually applying the electrodes but the simpler the method the better. With some apparatus the patient's resistance is measured before the fit is given. The patient's resistance gives no indication of the strength of the shock needed to effect a convulsion since living tissues do not obey Ohms' Law. Its chief value was to indicate the efficiency or otherwise of the circuit and since this can be done in other ways, the practice of measuring patients' resistance has practically dropped out. Resistance diminishes for three to four successive shocks but thereafter is little changed.

When the switch is pressed the patient gives a start as the current passes, and the fit follows at once. The course of the fit is similar to that described after chemical injection. If the current is insufficient a 'sub-shock' occurs. Some psychiatrists immediately give a further shock while others prefer to wait till breathing is reestablished and all chance of a delayed fit after Io to 15 seconds gone. The dosage can either be increased or merely repeated according to one's estimation of how nearly the fit.was missed. The initial voltage and time, or number of Joules, varies with different types of apparatus. One starts with the basal time and voltage recommended for the particular machine and if a subshock occurs the time may be increased by 0.1 second or the voltage by ten, or both. Patients who have many sub-shocks do less well clinically than those who largely avoid them, so that it is advisable to be fairly generous with the dosage and secure regular fits rather than give too little and have to repeat shocks. In a patient of average physical health one can give as much as three to four shocks in succession if the first three fail to cause a convulsion.

Dentures and hair clips must be removed before treatment, and the bladder emptied.

\section{Complications}

(I) Fatalities are certainly rare. Kalinowsky (1946) reported on 2,000 cases without a death. the author has known of one, due to coronary disease, in over a thousand patients, in which death could be said to have been hastened by the fit. Kolb and Vogel in a survey of all American hospitals found a death rate of 0.6 per cent. for E.C.T. and o.I per cent. for metrazol (cardiazol), while Impastato and Almansi found 0.8 per cent. in a survey of the literature on E.C.T. Unfortunately in many reports the actual connection between the treatment and death is poorly described and it is difficult to form an opinion from a statistical point of view of the risk entailed. One risk has to be balanced against another since the death rate from suicide is much higher amongst those depressions not treated by E.C.T. than amongst those so treated. Proper physical examination supported by $\mathrm{X}$-rays and other special methods if necessary will exclude the vast majority of conditions that can determine a fatal outcome. For comparison it is well known that uncomplicated single fits in idiopathic epilepsy are very rarely fatal.

(2) Fractures and dislocations. Collectively these are probably the most troublesome of all complications. The most common are compression fractures of vertebrae bodies although in I2 years' experience of the treatment no lasting functional disablement appears to have been recorded. The vertebrae most often affected are the $5^{\text {th }}$ to 8th dorsal with occasional fractures as high as the third dorsal and as low as the second lumbar. While the vertebral body may be uniformly compressed, a wedging is commoner with the narrow end of the wedge at the anterior border. The extra support of the pedicles seems to prevent the posterior part of the body being appreciably flattened as a rule. These fractures usually happen within the first five fits. The patient should be kept in bed for a fortnight and then treatment can be resumed if preventive measures as described below are used.

These fractures may be associated with no pain at all or with some soreness and tenderness to percussion over the vertebrae involved. Very rarely, if local swelling and oedema is sufficient to press on spinal nerves, there is referred pain round the sides of the chest to the epigastrium. Ir one of the author's patients it was severe enough to need morphia for a few days. Such pains always clear up with rest, with or without sedatives. 
Many patients complain of pains in the back, especially after the first few fits, but unless these conform to the types mentioned they have no diagnostic value regarding the presence of a fracture. Many complaints of pain in the back are really due to muscular soreness and cramp from the unusually severe exercise involved in a fit. Radiological diagnosis cannot be used to full advantage unless all patients are X-rayed before treatment, anteroposteriorly and laterally. In practice it is now considered unnecessary to do such routine $\mathrm{X}$-rays before or after treatment unless specially indicated. The incidence of these fractures has been estimated variously by several authors but the average is represented by the report of Cook and Sands (I94I) at I4 per cent. of male and 12 per cent. of female patients if no prophylactic measures are taken. The incidence is reduced to approximately 4 per cent., and the severity of the lesion is less if care is taken to give the fits with the spine moderately extended. With fracture boards under the mattress, a firmly rolled blanket is placed under the mid thoracic spine; one nurse exerts pressure on the shoulders over the heads of the humeri, another fixes the pelvis. In this way sudden flexion at the passing of the current, or during the fit, so liable to damage the arterior parts of the vertebral bodies, is avoided. Similar spinal fractures occur less frequently and without symptoms in epilepsy. None the less it is obviously desirable to minimize damage by extension of the spine as above.

The most serious fractures are those of the head of the femur. The author has not yet experienced one but they occasionally occur, even in both bones simultaneously. Elderly and very muscular younger patients are the most liable. Except for keeping the pelvis fixed, it is probably desirable not to restrain leg movements during the fit. Fractures of the acetabulum rarely have been reported. Fractures of the humeral neck and dislocations at this joint are recognized, and are especially liable to happen if the patient flings the arms outwards at the start of the fit. Dislocations are usually forward and if the shoulders are properly held during the fit, the head of the humerus may be felt thumping against the hand of the restraining nurse. It is advisable, therefore, to fold the arms across the chest and keep the bedclothes well tucked in at the sides so as to avoid any sudden excursion, but not so as to fix the arms completely.

Fractures of the scapula are rare. Dislocation of the jaw is perhaps the most frequent complication but is easily prevented and remedied. To prevent it, the jaw should be held firmly up from below. Some dislocations right themselves spontaneously in the later stages or at the end of the fit, and if not, the jaw can be easily replaced by hand, with the protection of a towel in the relaxed period at the close of the fit before consciousness is regained. Muscles, tendons and ligaments are rarely injured in convulsion therapy.

In practice, if care is used, these complications have not assumed an importance sufficient to cause discontinuing the treatment. By the intravenous injection of curare before each fit the risks of fracture or dislocation can be exluded, Bennett (1940). Such injections carry their own liabilities, so that most psychiatrists only use it in patients known to have a special risk of fracture or for the protection of an already damaged limb, perhaps from a suicidal effort.

(3) Respiratory complications. The chief difficulties are the apnoea already described, aspiration pneumonia and activation of quiescent pulmonary tuberculosis. It may be added that apnoea is more frequent after electrical than chemically produced convulsions, and more usual after sub-shocks than full fits. Aspiration pneumonia due to the inhalation of septic material more especially in the presence of dental sepsis is rare. Activation of latent pulmonary tuberculosis has been reported and, generally speaking, patients with quiescent and active pulmonary tuberculosis should not be treated with convulsion therapy. Any doubtful case should be X-rayed first and subsequent films taken during and after treatment. One cannot give an entirely hard and fast ruling on this point since there are times when a tuberculous patient who has become very depressed, and not taking food properly, may be benefited by E.C.T. His neglect of himself by self-starvation or attempted suicide may prove a greater hazard than the tuberculosis. Clearly one risk has to be weighed against another.

(4) Cardiovascular complications are occasionally encountered. Cardiac arrest with extreme pallor and widely dilated pupils has been seen and may recur several times in the same patient leading to cessation of treatment. It is thought to be due to cerebral vagal stimulation. Subconvulsive responses seem especially liable to provoke this phenomenon. Convulsions have at times expedited coronary thrombosis in those so predisposed. On the whole one has to consider whether the patient's cardiovascular system can stand the muscular exertion of the fit. If necessary do exercise tolerance tests, and use electrocardioraphy. Provided compensation is satisfactory there are many patients with cardiac lesions who can be treated successfully with E.C.T.

Electro-cardiographic changes so far reported, have not been striking, nor specially useful in deciding fitness for treatment. The most frequent abnormalities are minor arrhythmias, though 
they are absent in spontaneous epileptic seizures. Sinus tachycardia is followed by arrhythmia and extra systoles in the convulsive response, while in sub-shocks a bradycardia is common. This is thought to be due mainly to vagus stimulation which in the major fit is counteracted by the associated muscular effort. The author has seen a sub-conjunctival haemorrhage and others have been noted. These absorb without difficulty and treatment can be continued.

(5) The only neurological complication of note concerns the occurrence of spontaneous fits days or months after E.C.T. There has been much speculation as to the possibility of the treatment causing epilepsy in those in whom the disease was hitherto unknown. Occasional fits have been reported weeks or months after convulsion therapy but the view of the majority is that such fits only happen in those patients possessed of a predisposition to epilepsy prior to treatment. Such a predisposition can be shown by an electroencephalogram (E.E.G.) and no patient has been known to have fits after a course of convulsion therapy whose E.E.G. was formerly within normal limits.

(6) Psychiatric complications are few and are to be distinguished from those phenomena habitually present in greater or lesser degree in any patient treated by a course of fits. The latter are described under common forms of reaction to the treatment. One well recognized complication is the transformation of a manic depressive patient from depressed to manic phase by over frequent or lengthy treatment. Rarely activation of a psychosis may occur after a few fits, with an acute flare up of psychotic symptoms. As a rule the patient settles down again and after a week treatment can be continued. It is essential to differentiate this occurrence from the commoner organic reactions described later. Occasionally some patients, more particularly men, become excited during the half hour immediately following the fit. They may fling themselves out of bed or even attack those near. The intravenous injection of sodium amytal 0.2 to $0.3 \mathrm{gm}$. immediately before the induction of a fit generally prevents such events without stopping the fit.

(7) The electro-encephalogram shows changes resulting from E.C.T., though not if sub-shocks without a convulsion are administered. A progressive slowing of the normal alpha rhythm occurs until considerable delta activity is evident. Such changes depend on the frequency and duration of the E.C.T. and also on individual susceptibility. As a rule the more often fits are given and the longer treatment lasts, the greater the degree of dysrhythmia, though one patient may be much slower than another to show E.E.G. abnormalities. There is no correllation between the onset of improvement clinically and the changes in the E.E.G., though changes in memory and confusion parallel the E.E.G. more closely. E.E.G. abnormalities, like the defect of memory and confusion, are reversible, and disappear in two to eight weeks in the vast majority of patients. Those who have had most fits are slowest in recovery from dysrhythmia.

\section{General management of the treatment}

(I) When to begin ? For a suicidally depressed patient not under control it is necessary to begin treatment at once and repeat in a few hours if conditions of safety are still lacking. If a patient is refusing food, one to three fits on successive days generally ensures a resumption of feeding, and tube feeding may be stopped. Sometimes nutrition is so bad that artificial methods of feeding have to be used for a few days before the patient is strong enough to be treated. In the absence of these complications, there is little point in delaying treatment once examination is complete, since most patients have been ill for months before admission, and it is less than human to prolong their suffering unreasonably. Delay is only indicated if the diagnosis is doubtful, if the onse is very recent, e.g. a week or two, or if there is history of recurring short attacks from which spontaneous remission is likely. Successful treat ment is possible even after three years of illness:

(2) How often and how long to give treatment? Most depressions do well with two fits weekly, having perhaps three in the first week, with a total of four to ten. Depressions complicated by delusions tend to need more, and up to 15 are sometimes necessary. Schizophrenias require three fits per week and a total of 14 to 20 as a rule. Manias are best treated energetically at first with daily fits or two fits a day for three days, later spacing out to three a week as the acute phase is controlled.

(3) When to stop. When a depression is clear of symptoms two further treatments should be given before treatment is terminated ; similarly in schizophrenia, though more caution is needed in stopping the treatment of this disease. Depressions which are part of a manic-depressive psychosis need most careful observation for the earliest indication, usually failure to sleep, that may herald the onset of a swing into mania. It should be possible to prevent it by prompt termination of treatment and the use of sedatives.

Relapses. On this point it should be emphasized that convulsion therapy can cause remission of a psychosis but it confers no immunity to subsequent attack. For instance, those patients whose depression is of the recurrent type, con- 
tinue to have further attacks. Apart from this there are several factors liable to precipitate relapses. One of the commonest is a failure to discover factors that have played a dominant part in causing breakdown. If the patient is too incapacitated by depression to co-operate in a full enquiry prior to treatment, attempts can be made to complete the history and to search for the stresses or faults in the patient's way of life as soon as improvement begins to show itself.

Insufficient treatment. When improvement has set in, patients sometimes wish to break off treatment. It is usually unwise to do so before symptomatic recovery, followed by two further fits. Relapses and indifferent results are more likely in those depressions which occur in persons already subject to chronic neurotic symptoms, or having psychopathic personality, organic brain disease or schizophrenic features. Schizophrenia may begin with symptoms of emotional quality and when those are cleared by E.C.T. the essential schizophrenic nature of the illness is then shown.

The total relapse rate for the treatment is approximately 28 per cent., but if transitory and minor relapses are exluded, the figure is not more than 14 per cent. The majority of relapses occur within the first five weeks after the end of treatment, and observation should be maintained for this period. In practice many patients have to be treated psychologically or may need various social adjustments done for them in this time.

Out-Patient E.C.T. In view of the quick recovery many patients make from a fit, out-patient treatment is possible and often practical. It is indicated where the illness is mild and the patient fairly well controlled, and if it is desirable to keep the patient at work. Some involutional depressions and recurrent cases who have been treated, as inpatients on a former occasion, and whose reaction to E.C.T. is known, may be so treated. Manicdepressives in depressed phase should be excluded owing to the liability of a swing into mania. Because of the risk of confusional states especially in younger patients, not more than two fits a week are advised, though if as commonly happens only one fit a week can be managed treatment may prove inadequate. So far as possible, out-patient E.C.T. should be backed by the resources of a general hospital in case of complications. Patients are allowed to rest for an hour after treatment and should go home with relative or friend. On the whole, out-patient treatment is something of a makeshift, but is better than none at all. Owing to slight tension before, and confusion after the treatment, it is difficult to get a true estimate of the patient's condition if seen on the same day, and one misses the first hand observation and fuller interviews that go with in-patient care. It is difficult to vary frequency or spacing of treatment to individual needs and the procedure tends to become a very mechanical affair.

Psychiatric observations. Some phenomena are characteristic of all patients having convulsion therapy. There are two main lines along which improvement or recovery develops. The most desirable and specific is that shown by the recovering psychotic depression. In these patients a change for the better may be discerned after one treatment but more often after two or three. It is in the essentials of life that gain first appears, in sleeping and eating. More notice is taken of environment and there is less preocupation with self. Speech returns in those who were stuporose, and better attention is given to elementary hygiene. The patient becomes more accessible whether the depression was originally of retarded or agitated type. Questions are asked showing a suitable desire for reorientation, and the patient throws off the appearance of misery. Disorders of thinking - usually recover last, namely delusions of wrongdoing, guilt, disordered bodily function and the like. These changes occur at varying rates in different patients. In some it all appears to happen overnight, in others over a week or two. It is most impressive to encounter the normal personality for the first time when relieved of the havoc of a depressive psychosis. In some degree such a transition should be apparent in all well selected depressions.

While the foregoing picture is characteristic of the treatment response in emotional or affective illness, a different reaction is usual in illnesses that are primarily disorders of thinking; the schizophrenias, paraphrenic and paranoid psychoses. Most of these show no benefit until a mild temporary organic confusional state has been produced. To achieve this, it is necessary to give more frequent fits, at least three a week, for the longer periods customary in such cases. Confusion generally appears between the fifth and eighth treatments. Patients complain of loss of memory, of forgetfulness, have little capacity for cohnected thinking, are cheerful in a simple; easily satisfied way and apt to lose bits of property. Sometimes under the cloak of such confusion a lasting recovery may develop, but too often in one to three weeks after ceasing treatment the confusion clears and schizophrenic or paranoid symptoms reappear. Consequently, though individual patients do well, the results in the schizophrenias are little different from controls over large series of cases. It is characteristic of neuroses that they complain of memory loss much earlier than the psychoses, but with rare exceptions there is no evidence of lasting defect in memory or intellect in any type of patient. 
Psychological treatment. "While in numerous instances convulsion therapy is clearly the chief agent in recovery, and some depressed patients do well with little or no other assistance, there are others in whom psychotherapy is the other half of the necessary treatment. Particularly is this so in cases associated with illness precipitated by environmental stresses (reactive types), and in those complicated by neurotic features of hysteria or anxiety. It is not a question of a physical or a psychological approach to treatment. The methods are complementary and their use is regulated by diagnosis, and the progress of the patient. Most of those suitable for E.C.T. are not accessible to psychotherapy, and proper rapport with the physician is not obtained. After depression has been eased by E.C.T. patients are able to benefit from psychotherapy provided this is not attempted on the same day as a fit has been given, when concentration is temporarily impaired. In depressions secondary to neurosis, psychotherapy is the treatment of choice. It is also indicated in depressed patients who have been adequately treated with E.C.T., shown a remission, and then relapsed owing to reactive factors or personal difficulties hitherto unsuspected.

Results are similar whether the electrical or pharmacological method is employed. In depressions of manic-depressive psychosis, in involutional depressions and simple depressive attacks, recovery rates of 75 per cent. to 90 per cent. have been reported frequently. Investigation has even been made to ascertain why five patients out of a series of a hundred did not improve. It is known that these patients might eventually recover or improve spontaneously if untreated after from three months to a year or longer. About a third do not spontaneously recover. Nowadays mental hospitals contain few chronic depressions where E.C.T. is employed. Such as are still hospitalized are mainly those untreatable for physical reasons. Depressions that are allowed to continue untreated often remain exposed to suicidal and other risks. They are liable to lose their employment and have much difficulty in rehabilitating themselves if they recover later.

In mania results do not approach those seen in depression. Because the condition is so much less common than its depressive counterpart, large numbers of cases have not been reported. It is doubtful if convulsion therapy shortens the total time of the attack, but there is no doubt that a concentrated course of fits can bring the manic attack under control more safely and rapidly than narcosis unless the illness be of a mild type.

Schizophrenias. Cases of less than a year's duration often show favourable results but in all cases relapse is frequent, and over large series of patients the follow-up results are little different from controls. It is in the sub-groups of catatonic excitement and stupor that better results are obtained. The results in schizophrenia treated by convulsion therapy are inferior to those obtained with insulin coma treatment, but if the latter is not possible then E.C.T. should be given in the above clinical categories: Chronic schizophrenias can often be helped through their more difficult phases by repeated treatment over a long period.

Commentary. Now that a dozen years and more have elapsed since the first trials of convulsion therapy were made, some estimate of its place in psychiatry is possible. The method has been freely criticized though mainly on theoretical grounds. When given at the usual rate of two to three treatments weekly for a total of 12 or less, there is no evidence with the methods available at present, of irreversible pathological changes in the brain, and with much larger numbers of fits it is remarkable how little can be found. In animals subjected to convulsive states more severe than those given to patients, petechial haemorrhages were discovered, but confirmation has been lacking. Such haemorrhages do not appear after the much longer passage of a current $\left(7 \frac{1}{2}\right.$ minutes) in electronarcosis nor in the judicially electrocutecof Necropsy findings in deaths associated with con vulsion therapy have been meagre. In this respect the treatment differs strongly from the pathology noted after insulin coma deaths. The brain is known to be able to support an amount of current far in excess of that commonly used for E.C.T. In practice it is better to give ten volts too much or a time of 0.2 second too long than have many sub-shocks. Technically the treatment is simple and does not need any great knowledge of electricity. Complications are few when it is carefully administered. The skilful part of this treatment is not in 'pressing the button' but in painstaking examination preceding its use, in psychiatric interviews between treatment, deciding how often to treat and when to stop.

The rationale of the treatment is not known. Metabolic, biochemical, diencephalic, pavlovian and psychological mechanisms have their advocates.

The interesting work of Masserman and Jacques on electro shock and experimental neurosis in cats supports the view that the treatment produces a certain amount of disintegration so far as complex neurotic patterns, such as phobias, compulsions and inhibitions are concerned. It is then possible for latent and more normal patterns to reestablish themselves, for new forms of adaptive behaviour to arise, and for re-education to pro- 
ceed. They also remark that the "price of recovery' was a variable loss of certain higher adaptive capacities.' Some of their animals had not regained their normal efficiency in complex skills of which they had formerly been capable by the end of the post shock testing period.

With patients, clinical observation suggests a similar process goes on, but the recuperative powers of the brain are such that the majority of those who are considered recovered reach a level of efficiency which, to near relations, is the same as prevailed before breakdown. The Rorschach and the Minnesota multiphasic personality inventory tests were employed by Pacella, et al., 1947, to assess the effects of E.C.T. on some aspects of personality in 75 psychiatric patients of schizophrenic, manic depressive and psychoneurotic types. The Rorschach results of successful cases showed that basic personality defects remained after treatment, but that thought, emotions and action were under better control, and more efficient use of remaining capacities was made. There was no gain in intellectual capacity in schizophrenias but improvement in intellectual efficiency was notable. The Minnesota test was not specially useful except in determining if improvement in schizophrenia after treatment would be retained.

\section{Insulin Coma. Treatment}

It is 15 years since Sakel made his first report on the treatment from the University Clinic of Vienna. The 1939-45 War caused the closure of numerous insulin units in this country, but many have since resumed activity. Although long term results are open to criticism, the method remains unique in being the only one known to regularly cause schizophrenic patients to change for the better, if one excludes certain types treated by leucotomy. In view of the chronic nature of many schizophrenic illnesses, this observation is obviously of cardinal importance even if it falls far short of perfection. The treatment is particularly helpful in improving the disordered thinking of the schizophrenic patient. Any influence it may have on emotional disturbance is probably secondary to this. It is not useful in manic depressive and other psychoses where emotional disorder assumes the primary role.

Selection of patients. Schizophrenias are the only group of psychiatric disorders in which insulin coma therapy is widely used, and the decision to give or withhold treatment is made after considering the following factors :-

(1) Type of schizophrenia. The most suitable patients are the paranoid schizophrenias, followed by the catatonias ; while simple and hebephrenic types are the least amenable to treatment. Many patients are not easily classified in these main categories and are termed 'atypical 'because of manic, depressive, hysterical, obsessional or other complicating features. Many such cases have responded well. There is little evidence that insulin substantially helps those who can truly be labelled Dementia Praecox, where dementing processes set in during adolescence and progress steadily to complete invalidism. Fortunately such cases represent less than Io per cent. of the whole schizophrenic group, though formerly the term was applied incorrectly to all schizophrenias. Patients having this malignant form of schizophrenia are most likely to be found amongst the simple and hebephrenic types of more recent classifications.

(2) Duration of illness is perhaps the most important single consideration. All statistics agree that cases of a year or less show substantially better results than those of longer duration. After two years few patients can be benefited by the treatment. Thus while several months delay does not of itself vitiate the result of a depressive psychosis treated by E.C.T., a similar delay in schizophrenia is likely to be fatal to a patient's recovery with insulin. For this reason Sargant and Slater remark that early cases of schizophrenia should have high priority on waiting lists. If careful enquiry is made, it is generally found that the disorder has lasted longer than was first apparent. The presence of previous attacks, especially if followed by satisfactory remissions, is no bar to treatment and may be associated with good prognosis.

(3) Type of onset. The slow insidious onset, lacking any precipitating cause, is unfavourable for treatment and prognosis; while those who suddenly fall ill, showing a wealth of symptoms, with a history of severe precipitating stress, generally react well.

(4) Prepsychotic personality. There is no doubt that the chances of a successful outcome are increased in those patients known to have shown a healthy adaptation to the difficulties of life before breakdown. Such patients are generally found to have been reasonably successful in their occupational, home, social and other personal relationships. By contrast, treatment is unfavourably influenced in those whose history makes it clear that they have been shy, diffident types from an early age, prone to circumvent and avoid awkward situations when a solution is necessary. They do not mix well, form few friends, and are apt to achieve their ambitions in day dreaming rather than in reality. From a mental background of this nature, schizophrenia is liable to develop in- 
sidiously, and the patient's illness appears as an almost logical development in a predisposed personality. While insulin treatment may arrest the process towards a seclusive fantasy life, there is little reason to suppose that constitutional trends of this nature can be radically altered. Similarly, those hampered by intellectual defect, psychopathic or neurotic qualities in the personalty have a less favourable chance with treatment than in the absence of such traits. In line with the foregoing is the view that the extravert personality reacts better than the predominately introverted, and of physical constitutional types the pyknic and athletic habitus are more favourable than the asthenic or dysplastic.

(5) The factors of heredity, age and sex are of less value in assessing the outcome. Hereditary tainting in near relations was thought to be a disadvantage but a number of cases thus handicapped have done quite well. Generally speaking, the results are poor in patients under 18 years of age and after 40. The former appear to have little resistance to the schizophrenic process and views are conflicting regarding the late age groups. There is no substantial difference between the sexes as to results.

Risks and contraindications. In the hands of experienced insulin therapists, risks are no greater than are accepted in other branches of medicine. In large series of cases collected from several hundred hospitals in the U.S.A., mortality figures varied from I.29 to 0.85 per cent. (Kalinowski and Hoch, 1946). Mortality figures are usually higher in surveys covering numerous hospitals and the figure is not more than 0.5 per cent. in the most skilfully treated series. The cause of half of all deaths is hypoglycaemic encephalopathy after irreversible coma, which is largely an avoidable risk. The remainder are mainly due to aspiration and other forms of pneumonia, and to cardiovascular disturbances.

Despite these reports the risks of treatment are on the whole less than the risks of waiting for a spontaneous remission to occur. The figures of New York State hospitals show a higher mortality amongst the untreated compared with those treated. The untreated not only carry the risk of mental invalidism but of physical deterioration, proneness to tuberculosis and intercurrent illness. Twelve patients died in a control group compared with one in the treated series.

Mentally the chief risk is of a mild degree of intellectual impairment where long series of deep comas are required. Only a small number of patients ever show this feature and it is probably less than a similar impairment seen in convulsion therapy. Greater degrees of intellectual deficit are seen after irreversible comas, when a Korsakow type of picture is common. It usually improves though recovery is not always complete.

Contraindications are on advanced degree of $\frac{\Phi}{\varrho}$ cardiac disease, renal and liver diseases, diabetes $c$. mellitus and thyrotoxicosis. Treatment should be $\underset{\vec{*}}{\vec{F}}$ suspended for any fibrile illness.

Technique of treatment. Although suitable literature is available (Sargant and Slater, $1948 ; \frac{\bar{c}}{\bar{c}}$ Kalinowski and Hoch, 1946), the acquisition of $\frac{\infty}{\sigma}$ sound technique is an essentially practical matter. $\stackrel{\circ}{2}$ On this account and in view of the necessary $\%$ limits of this article, it is proposed to deal mainly $\vec{O}$ with some observations which further experience:of insulin in both general and mental hospitals $\vec{\omega}$ suggest as important. From a purely technical point of view insulin coma treatment demands 0 far more skill from both medical and nursing staff than does convulsion therapy. Preliminary experience in an active insulin unit is desirable before embarking on treatment for the first time. + It has been shown that the difference between the expert and the indifferent insulin therapist can ${ }_{0}$ mean as much as 30 per cent. in the recovery? rate after treatment. Generally speaking, six cases is the maximum that one doctor can manage and a colleague must be on call. The situation is $\frac{0}{5}$ similar to looking after six general anaesthetics at $\stackrel{\text { ? }}{\rightarrow}$ the same time for five or six days a week. T Te $\vec{\varphi}$ same therapist should be in personal charge for spo months to a year or more and frequent changes $\overline{\text { fof }}$ medical or nursing staff are to be avoided. The nursing responsibility is a matter for a senior member of the nursing staff experienced in the treatment. She should not be liable for any duties $\mathbb{\Omega}$ outside the insulin room during the morning. Two other nurses in training assist her and a fourth is $\frac{0}{3}$ available if required. It is advisable for the senior $?$ nurse to be unchanged for nine to 12 months. In wards with patients under insulin treatment, it is occasionally necessary to deal with manifestations 3 of hypoglycaemia during the rest of the day or: night, so that staff and apparatus to combat signs? of relapse must be available. For this reason, patients are not dispersed to other wards after the $₹$ treatment and are only allowed out for shorto distances if accompanied by a nurse carrying an emergency ration of sugar or sweets.

The main routine of treatment has altered little since Sakels' original outline was published. In-O sulin is injected intramuscularly at 7 a.m., then patient having fasted since supper time on the N previous evening. If intravenous insulin is used. the injection time is 9 a.m. The initial dose is usually 20 units, increasing by 1o units daily until 90 units has been reached, and then by 20 or evence 40 units a day until sopor occurs, when the increase may be slowed. During the first twoo hours patients lie dozing, and towards the end of 
this time begin to flush and perspire. Some get very hungry and may attempt to get food from lockers. Between 9 and 9.30 a.m. the stage of sopor is reached, and is recognized by considerable impairment of consciousness, loss of orientation, of speech and co-ordinated thinking. Most patients sleep quietly through this phase though they still react to painful stimuli and corneal reflexes are preserved. Some become very restless, excited and noisy, and need restraining temporarily. Between I0.I 5 and I I a.m. sopor passes over into the true coma stage. This is recognized by the patient being completely unconscious, by the lack of any purposive response to stimuli, such as pricking or pinching, by the absence of corneal reflexes and by a positive Babinski plantar response. While it is essential that loss of consciousness and purposive response be considered criteria of corma, the other signs are apt to vary in the time of their appearance and one should not necessarily wait until all are present until diagnosing coma. The phase of coma has to be achieved for the best therapeutic results. On the first day that coma occurs, it is interrupted at once. On succeeding days the comas may then be increased by five minutes a day until 20 minute comas are reached. Longer coma periods are possible, but at the expense of additional complications, and lacking additional benefit to the patient. If the dose has been insufficient to cause coma, patients should be interrupted after $1 \frac{1}{2}$ hours of the sopor phase.

Once coma has been established, patients tend to become increasingly sensitive to insulin, and coma comes on earlier. Dosage should be cut after the first coma so that patients do not go into coma earlier than I0.15 a.m., and the decrease is continued until it is clear that the minimum necessary has been attained. In practice it is commonly found that the patient's usual coma dose is as little as half the initial coma producing dose. Neglect to allow for the phenomenon of insulin sensitivity is one of the chief causes of the complication of irreversible coma.

Danger signs. During the coma period, twitching is often seen, usually round the mouth at first. In a few patients it increases to involve the whole body and precedes a full convulsion. If there is a fit, the patient may be partly wakened up by it. Fits may also occur before the coma stage, but are easily managed and subsequently controlled by the use of anticonvulsants. In any event, treatment should be interrupted. Waves of extensor tonus occur in deep coma, with increased tension of all muscles, extension and pronation of the arms, increased rate and depth of respiration, and dilation of the pupils. Though separated by intervals of relative placidity, they tend to increase in frequency as coma deepens, and are most exhausting. Circulatory failure is indicated by fall in blood pressure and by discoloration of the finger tips together with extra systoles and pulse irregularities. Pulse rates below 55 make interruption advisable, as does persistent tachycardia above I Io or a blood pressure below Ioo. Occasionally great restlessness and excitement in early coma make treatment difficult and may be avoided by the use of intravenous insulin or premedication with sodium amytal, gr. 6, at 9 a.m. Excessive salivation sometimes embarrasses respiration. The head should be turned to one side and atropine, gr. I/100, given at 9 a.m. for subsequent comas.

Most patients have their own distinctive pattern of sopor and coma. Although fits, extensor tonus and spasms, and circulatory failure are indications for interruption in any patient, there are many lesser individual variations that are significant in some patients and not in others, or are only significant at certain stages. The insulin therapist who knows the individual patterns for each case gets to know when a change from the usual course means danger. It is necessary to deal with complications as early as possible otherwise poor gastric absorption or collapsed veins may render interruption difficult.

Interruption of coma is accomplished by giving glucose either through a nasal tube or intravenously. The nasal tube is inserted shortly before coma should cease and its passage to the stomach confirmed by testing gastric juice against litmus paper. As much gastric juice as possible is sucked out to avoid dilution of the sugar solution or over distension and vomiting. At the interruption time $600 \mathrm{cc}$. of 33 per cent. glucose, warmed and flavoured with lemon or tea, is poured down. Slightly less may be used for females. The flavouring is for the benefit of patients who may need a glucose drink when still in a conscious state. Many comas are terminated regularly by this technique, but if after $I_{5}$ to 20 . minutes the depth of hypoglycaemia is not substantially diminished, then intravenous interruption is given at once.

This method of termination is always used by some psychiatrists as being more certain and therefore safer than the nasal route. Others prefer to employ it only in the event of failure with the other method. From roo to $200 \mathrm{cc}$. are given, and the patient usually recovers consciousness in a few minutes. It is important to note the time taken for recovery since if recovery is delayed after I0O cc. of glucose has been given, the likelihood is that too much insulin has been 
administered, that an irreversible coma is near, or that coma has been allowed to go on toolong. The simplest way to give the glucose is by a simple pressure apparatus after the type originally used at the Maudsley Hospital by Sargant and Fraser. A graduated bottle of $500 \mathrm{cc}$. capacity is fitted with a two way cap. One opening is connected by rubber tubing with a pressure bulb, the other is connected by tubing to a syringe with a side opening situated one-third of the way from the needle end. The pressure is raised and the system filled with glucose and, with the plunger closed, the vein is entered as usual. The plunger is withdrawn and as it passes back past the side opening, the pressure forces the glucose over, and all that is. needed therafter is an occasional squeeze of the bulb. The system is very adaptable since the pressure bottle can be unscrewed and replaced with the same or a different solution as required. After intravenous interruption alone, awakening is followed by a light meal of sugared tea and sandwiches. For the rest of the day it is essential that the patient takes a full lunch, tea and supper, but no special loading of the diet with carbohydrate or anything else is necessary. To give extra carbohydrate is merely to increase resistance to insulin next day. Ideally the carbohydrate intake should be kept constant, but for various reasons it is generally not possible to achieve this in psychiatric patients.

Patients who have had insufficient glucose at the interruption time, or who are difficult with their food, or in whom large injections of insulin have been needed, are liable at times to show signs of late hypoglycaemia in the afternoon. If proper observation is maintained so that a drink of glucose or sugared tea is given in good time, there is generally little difficulty in restoring the situation. None the less, a tray. equipped for intravenous interruption must always be ready for use in emergency on the insulin ward.

The total number of comas required depends on how soon improvement begins, and may vary from 30 to 60 . It is unwise to exceed this figure because of risk of damage to the brain.

It is essential that the time of onset of each phase, sopor, coma, etc., should be noted as these occur, and a time for interruption ordered as : oon as coma is diagnosed. Only by accurate timing can the pace at which hypoglycaemia is proceeding be ascertained, and on this the safe length of coma decided daily individually.

Irreversible coma. This is one of the most dangerous complications of the whole procedure, though one which is nearly always avoidable. It is considered to be present when the patient fails to recover consciousness after 250 cc. of 33 per cent. glucose intravenously. If a nasal feed is followed by facial pallor, vomiting and non absorption by the stomach, there seems to be special liability to the irreversible state. Someo patients have a tendency to irreversibility from the start of comas and are slow in reacting to glucose intravenously. Most irreversible comas occur in the sensitization period early in the coma series: through failure to reduce the initial coma pro $\overline{-\bar{s}}$. ducing dose quickly enough. In the early stage of irreversibility, the patient is usually restless, over breathing, flushed after initial pallor, and in aw shocked state. Treatment is directed towards $\overrightarrow{0}$ minimizing shock, maintaining nutrition and regaining consciousness. The patient is thereforew kept warm, the foot of the bed a little raised ando 2 cc. of coramine given intramuscularly. The intravenous glucose, 33 per cent., is continued until $400 \mathrm{cc}$. has been given. If there is much + restlessness, from 0.1 to $0.3 \mathrm{gn}$. of sodium amytal is injected through the tubing of the intravenous apparatus. Cyanosis and hyperpnoea are relieved by the intermittent administration of oxygen viao a B.L.B. mask. Most cases tend to settle down? after the first hour, but unconsciousness may $\vec{\not}$ continue as long as four to five days. In earlyo years cases were lost through what is now realized ${ }_{\mathscr{S}}$ to have been inadequate treatment, subsequently it has been evident that the same result may follof over enthusiastic efforts. Many cases reacted. ${ }^{\circ}$ well to $750 \mathrm{cc}$. of 33 per cent. and $1,500 \mathrm{cc}$. $4 \mathrm{f}$ 5 per cent. glucose spread over the 24 hours, given in three or four injections. In some patients the circulation could hardly support this amount and there was a tendency to pulmonary oedema. $\mathbb{Q}$ Latterly the author has dispensed with the use of $\overrightarrow{\overrightarrow{0}}$ 5 per cent. glucose, and while giving the same 3 amount of 33 per cent. glucose as before, has followed the glucose injections by $100 \mathrm{cc}$. of 500 per cent. sucrose, three times a day. This was successfully injected despite its high concentration, and after each injection patients certainly appeared to improve clinically and the comas to run a less 3 . malignant course. Apart from the foregoing there are few remedies that appear to have any decisive effect on the coma with the possible exception ofo blood transfusion. The vitamin $B$ complex is $>$ theoretically of value in facilitating cerebralo carbohydrate metabolism, and $20 \mathrm{mgm}$. of vitamin $\mathrm{Br}$ and $100 \mathrm{mgm}$. of nicotinic acid should? be given four hourly. Eucortone and calcium ${ }_{0}$ chloride have also been tried, as have many other N substances.

After the first two hours 4 fluid oz. of 330 per cent. glucose are given two hourly by stomach $\mathbb{\Phi}$ or nasal tube. When it is clear that these feeds? ${ }^{?}$ are being retained and gastric absorption resumed, $\frac{T}{0}$ then less intravenous feeding is required, though it $\frac{\text { }}{\mathbb{D}}$ should not be discontinued until eonsciousness is $\stackrel{\mathbb{P}}{\oplus}$ 
regained. On recovery, the patient is left with a varying degree of confusion and disorientation, and a mood that is cheerful, over placid and somewhat fatuous. They have few ideas and are easily pleased. They show little of their former illness. The confusion clears over the ensuing two to three weeks, depending on the length of the irreversible period. Comas may be built up again after a week's interval.

Psychiatric changes induced by the treatment. In promising cases there is generally temporary improvement for an hour or two after interruption. The patient achieves some.appearance of normality, their mood is more natural and they are more in touch with those about them. In this period they are most amenable to psychotherapeutic enquiry and treatment, and better rapport is possible. At this time too some are very dependent, seek reassurance, and are at pains to identify familiar faces and surroundings. In improving cases this easing of the psychosis becomes more permanent as further comas are given. On the whole few patients do well who have not shown some gain before 25 comas.

Convulsion therapy is sometimes combined with insulin, and if carefully used in selected cases the recovery rate of any series of schizophrenias is better than with insulin alone. Convulsion therapy may be applied two or three times a week in late sopor or early coma followed by immediate interruption, or given on a noninsulin day. The former is the most effective as a rule. The combined treatment is indicated where emotional disorder is evident, where there are severe catatonic features, or when a case has failed to improve after 20 or more comas.

Results from large series of cases show a three to one ratio of recovery and improvement in favour of insulin for those treated with two years onset. Statistics for New York State hospitals showed that this was maintained for two years, and 60 per cent. of treated patients were still at home in a recovered or improved condition. As after spontaneous recovery, relapses occur and with the passing of years treated and control cases tend to approximate, though according to Hinko and Lipschutz, after five years the remission rate is still 18 per cent. higher for insulin treated patients than in those allowed to remit spontaneously. Relapses can be re-treated, and the years spent out of hospital are a great gain for the patient, not to mention economic benefits to patient and community.

For patients treated in the first year a saving of 422 hospital days per patient was obtained, and remission permitting parole occurred in one-third the time required for spontaneous remission.

Modified insulin treatment. Insulin has one other useful place in psychiatry although indications and technique differ entirely from insulin comas. The principles underlying modified insulin therapy were embodied in the ideas of Weir Mitchell, but the present modified insulin method was first described by Sargant and Craske on the treatment of War Neurosis in 194I, and by the author in 19.44 in civilian neurotic states.

Many neuroses and some psychotic depressions deteriorate physically and lose weight on account of prolonged mental tension arising out of the conflicts of their illness. The decrease in physical well being renders patients even less capable of adjusting to their neurosis, and a vicious circle is set up. Restoration of weight in these neurotic cases enables many to renew their efforts at adjustment and to benefit more rapidly from psychotherapy. The treatment also has a mild sedative effect. It 'ts therefore not specific for any one psychiatric state but frequently proves to bo the turning point in a down hill course of illness and facilitates progress with the main psychotherapeutic procedure. It emphasizes the value of treating the whole patient and not being exclusively concerned with one aspect of their constitutions. That the good effect is not merely the result of suggestion has been shown by the lack of response to control injections of sterile water.

Insulin is injected intramucsularly at 7 a.m., starting with ro units and increasing by 10 units daily until a state of mild hypoglycaemia is evident between 9 and 9.30 a.m. The hypoglycaemia should not be allowed to go further than the stage of flushing, sweating and slight drowsiness, not amounting to light coma. To attain this phase may entail a dosage of from 10 to 100 units according to sensitivity. At 9.30 patients are roused for breakfast consisting of the menu for the day together with 8 to $\mathbf{I 2} \mathrm{oz}$. of mashed potatoes. Such is their appetite that this large meal is taken well as a rule. Any patient threatening to slip into sopor before 9.30 is aroused at once with a glucose drink and given breakfast. Though rarely needed, a stomach or nasal tube should be at hand for the administration of glucose in emergency. The increase in appetite often persists through the rest of the day. The patient is weighed before treatment and at weekly intervals afterwards, each time in similar clothing. Treatment is continued six days a week until definite gain in weight ceases, usually after four to seven weeks. How much weight is gained depends on the quantity originally lost. Gain at the rate of a pound per day is not uncommon. The treatment will not force patients above their normal weight, and those who are chronically thin are not fattened.

Indications. In general terms these are :- (I) To accelerate recovery in anxiety states, in hysteria 
and particularly in neurasthenic conditions of exhaustion or irritable weakness. At times these cases also show suspicious paranoid, mildly hostile trends, and it is difficult to make adequate rapport with the patient. It is remarkable how the sense of well being acquired under two weeks insulin can swing this attitude towards one of cooperation and subsequent progress with purely psychological methods. (2) To aid convulsion therapy in the treatment of depression and in combination with prolonged sleep for acute anxiety neurosis. The treatment is only of value where the foregoing clinical states are associated with metabolic change producing weight loss. Seven to ten pounds loss is the least change worth considering as significant.

\section{BIBLIOGRAPHY}

BENNETT, A. E. (1940), F.A.M.A., I14, 322.

CERLETTI, U., BINI, L. (1938), L'ELETTROSHOCK, Arch. Gen. di neurol. psichiat., e Psicoanal, 19, 266.
COOK, L. C. and SANDS, D. E. (1941), four. Ment. Sc., Vol. 87, No. $367,230$.

COOK, L. C. (1944), 'Convulsion Therapy, Recent Progress in $\frac{\widehat{D}}{\mathrm{Q}}$ Psychiatry.' Ed. by G. W. T. H. Fleming. London.

HINKO, E. N. and LIPSCHUTZ, L. S. (1947), Amer. Four.C

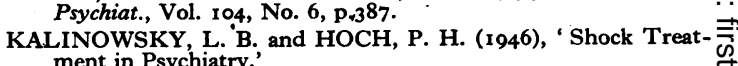
ment in Psychiatry.'

KOLB, L. and VOGEL, V. H. (1942), Amer. Four. Psychiat,, 99,.С 90.

MASSERMAN, J. H. and JACQUES, M. G. (1947), Amer. Four. Psychiat., Vol. 104, No. 2, 92.

MAYER GROSS, W. and WALK, A. (1938), Lancet, I, p. 1324.

MAYER GROSS, W. and WALK, A. (1939), Lancet, r, p. 1324.

MILLIGAN, W. L. (1946), Lancet, 2, 516, Oct. 12.

NEUSTATTER, W. L., LOND, M. B and FREEMAN, H. (1939), Lancet, 2, 1071.

OWEN T. V. and STEMMERMANN, M. G., Amer. Four. $\rightarrow$ Psychiat., Vol. 104, No. 6, p. 410.

PACELLA, B. L. PIOTROWSKI, Z. and LEWIS, N. B. C.S (1947), Amer. Four. Psychiat., Vol. 104, No. 2, 83.

SANDS, D. E. (1939), Lancet, I1, 250.

SANDS, D. E. (1944), Four. Ment. Sci., Vol. 90, No. 380, p. 767 그

SARGANT, W. and SLATER, E. (I944), 'Physical Methods of 'Treatment in Psychiatry,' Edinburgh.

SARGAN'T, W. and CRASKE, N. (194I), Lancet, 2, 2 I 2.

TOW, P. M. (1947), four. Ment. Sci., Vol. 92, No. 392, 644.

\section{BOOK REVIEWS}

\section{INTRODUGTION TO MEDIGAL PSYCHOLOGY}

By L. Erwin Wexberg, M.D. William Heinemann. 1948. Pp. 171. Price 17s. 6d.

The problem of teaching psychology to medical students has come to the fore of recent years. The interim report of the Royal College of Physicians (1943) stressed the need for instruction in normal psychology in the pre-clinical years, indeed 'the object of training at this stage should be to produce a good general practitioner and not a specialist in psychological medicine.' It was recommended that this course should be followed by an introductory course on the psychiatric aspects of clinical work at the beginning of the student's first clinical year.

Ebaugh and Rymer in their comprehensive treatise on Psychiatry in Medical Education (1942) are fully alive to the difficulties in teaching : "It is too little recognized that educators often do not know what to teach, how to teach, or even why it is necessary to teach such a course.'

The recent report by the B.M.A. on ' The Training of a Doctor' advocates the need for instruction , in normal psychology for the pre-clinical student, but deplores the absence of suitable textbooks in psychology for medical students. Wexberg's little book 'Introduction to Medical Psychology' certainly aims at meeting such a demand. The book is the result of the author's trial and error in methods of teaching psychology to medical students over a period of eight years.
While admittedly the book is ' an introduction,' it is a matter for regret that there is not more of it. The subject matter is conveniently divided into six chapters, at the end of which there is a useful list of $\stackrel{0}{\circ}$ references, to enable the student to undertake wider $\stackrel{1}{2}$ reading. The book is thoroughly readable and fills $\vec{\overrightarrow{ }}$ a useful gap not only for the medical student, but for 3 general practitioners, many of whom never had the opportunities provided today in the medical curriculum.

W.D.N.

\section{ELEMENTS OF SURGICAL DIAGNOSIS}

By. Sir Alfred Pearce Gould, revised by Sirô CeCIL P. G. WAKeleY, K.B.E., C.B., F.R.C.S. 9th Edition. Cassell. 1948. Pp. xv +718 을 Price I5s.

This excellent little book appears once again having now reached its ninth edition. Practically $N$ everything which the surgeon need know in os diagnosis is to be found within the covers of this $N$ pocket volume and though there are few illustra- N tions, the text is so clear that even these could be omitted. This manual of signs and symptoms has always remained quite distinctive, its appeal to the student being its completeness and accuracy. It is, $\mathbb{\Phi}$ in fact, the ideal reference book for ward and out-? patient department and should be carried in the $T$ student's pocket at all times. If it could be printed on India paper it would indeed be perfect. 